\title{
Educación, cibercultura e inteligencia colectiva
}

\section{Education, ciberculture and collective intelligence}

\author{
Fulgencio Sánchez Vera \\ Universidad de Murcia \\ f.sanchezvera@um.es
}

\begin{abstract}
RESUMEN
El ciberespacio está abriendo nuevos horizontes para la educación. Sin embargo, las posibilidades de interconexión, organización y creación colectiva que el nuevo espacio permite no siempre son entendidas y aprovechadas. Las administraciones educativas continúan promoviendo sistemas basados en principios predigitales para apoyar a las formas educativas tradicionales. Frente a ellos, se extienden otros que aprovechan los principios del ciberespacio desde nuevas creencias y valores, permitiendo formas de autogestión del saber y el conocimiento que están suponiendo una extraordinaria expansión de nuestra inteligencia colectiva. Consideramos fundamental replantear el encuentro TIC-educación bajo nuevas categorías que nos hagan tomar consciencia de la relevancia de esta visión y reorienten las acciones para la construcción de la escuela de la sociedad de la información.
\end{abstract}

\section{ABSTRACT}

Cyberspace is opening up new horizons for education. However, the new possibilities of interconnection, organization and collective creation are not always understood and exploited. The educational administrations continue to promote systems based on pre-digital principles as a support to traditional educational models. As opposed to these, other systems are extending the principles of cyberspace from new beliefs and values, allowing new forms of self-management of knowledge, which are resulting in an extraordinary expansion of our collective intelligence. We consider it essential rethink the ICT-education meeting under new categories that make us aware of the relevance of this vision and refocus actions for developing the school of the information society.

PALABRAS CLAVE

educación | ciberespacio | cibercultura | inteligencia colectiva | enseñanza-aprendizaje.

KEYWO RDS

education | cybers pace | cyberculture | collective intelligence | teaching and learning

\section{Introducción}

En las últimas dos décadas, las tecnologías digitales (1) se han convertido en tema de atención preferente en educación. La política, las empresas, el mundo académico y la comunidad educativa están dedicando importantes esfuerzos a su comprensión, desarrollo y aprovechamiento. Aunque cada actor tiene su propia agenda y actúa bajo intereses particulares, existe un discurso explícito compartido: se requiere preparar a los ciudadanos para la sociedad de la información y aprovechar las tecnologías digitales para mejorar la educación. Sobre estos principios se han movilizado el resto de actuaciones; no obstante, existen una serie de preguntas que es importante plantear: ¿Qué tipo de sociedad de la información nos orienta? ¿Qué tipo de educación queremos? ¿Qué formación requiere el ciudadano de hoy día? ¿Cuál es el potencial de las tecnologías? Cada actor tiene su propia visión; la diversidad se sustenta en posiciones ideológicas, intereses particulares, inercias y, especialmente, en la concepción que tenemos sobre las tecnologías digitales y las realidades que están generando. Estamos convencidos de que muchos de los esfuerzos que se realizan parten de una deficiente comprensión de la nueva realidad.

La importancia de este hecho es radical, pues la dirección del cambio cultural y el tipo de sociedad deberían sustentarse en un conocimiento profundo sobre las nuevas posibilidades de interacción y comunicación que están emergiendo. No podemos ignorar la importancia que ha tenido en la historia humana la aparición de tecnologías que potenciaban la comunicación. De hecho, delimitamos las fases históricas atendiendo a términos como cultura oral, cultura escrita, cultura impresa y, ahora, cultura digital. Cada una representa un hito en la comunicación humana, en las posibilidades de transmisión cultural y en el desarrollo de una nueva cultura y sociedad condicionada por el nuevo medio. 
Evidentemente, cada sociedad desarrolla nuevas tecnologías o asume las posibilidades de las existentes dentro de sus parámetros culturales. Tecnología, cultura y sociedad son elementos íntimamente relacionados que evolucionan desde la mutua interacción en un proceso continuo de cambios que, en algunos casos, pueden suponer sustanciales transformaciones para algún ámbito de la vida como puede ser la educación. En la actualidad, la interacción con los nuevos dispositivos tecnológicos está abriendo nuevas y fascinantes posibilidades para el aprendizaje individual y en grupo que están alterando las formas educativas tradicionales. Los cambios que se están viviendo en la enseñanza obligatoria son coherentes con los principios generales del discurso, orientándose fundamentalmente al desarrollo de la competencia digital del alumnado, a la mejora de los procesos de enseñanza-aprendizaje, y la comunicación entre los integrantes de la comunidad educativa. Las concreciones de estos objetivos pasan por modificar el currículo, dotar de medios a los centros, desarrollar nuevas competencias para el profesorado y crear unas condiciones que hagan asumibles las nuevas demandas y el cambio.

Un denominador común de las actuaciones llevadas a cabo es su orientación al aprovechamiento de los recursos, contenidos, herramientas y aplicaciones del ciberespacio para mejorar la clase y la escuela. No cabe duda de que se ha hablado de construir redes, de abrir la escuela a la participación de otros, de creación colaborativa, etc.; pero, sobre estos aspectos, los esfuerzos han sido comparativamente inferiores y, por supuesto, han tenido un bajo impacto en los centros.

Tradicionalmente, la investigación educativa se ha preocupado de analizar el nivel de presencia de las TIC en el entorno escolar, los efectos en el rendimiento y aprendizaje del alumnado, las expectativas, opiniones y actitudes de los agentes educativos (Cabrera 2013); y, sobre todo, se ha hecho un gran esfuerzo por divulgar las mejores prácticas de uso de las TIC en los centros y en las aulas, con lo que se ha generado un extenso banco de referencia sobre "casos de éxito" y "buenas prácticas". Sin embargo, es escasa la investigación centrada en el análisis, diseño y uso de sistemas para educación orientados a la interacción, comunicación y creación colectiva más allá de cada centro. Generalmente, se da por bueno la reutilización o agregación de herramientas y servicios creados con otros fines y para otros ámbitos, o el desarrollo de sistemas que suponen grandes inversiones, pero que son concebidos y gestionados bajo esquemas predigitales.

Consideramos que, en estos momentos, el conocimiento es aún escaso y las soluciones aportadas se han basado en la prueba y el error, por lo que no existe un suelo firme y la experiencia acumulada tiene mucho de tentativas sin continuidad. De todas formas, observamos elementos y tendencias que orientan realidades distintas, por lo que resulta de máxima relevancia investigar los sistemas que están contribuyendo al desarrollo del ciberespacio y la educación, especialmente en la generación de bienes comunales o procomún, y analizar cómo los actores educativos están actuando en su desarrollo. Para ello, partimos de algunos principios consustanciales al ciberespacio y valores altamente aceptados con el objetivo de definir una posición sobre su construcción que nos aporte un marco de referencia para el análisis y la interpretación de lo que se está haciendo en educación.

\section{Ciberespacio y cibercultura}

En estos momentos iniciales del desarrollo de las nuevas vías de adquisición-transmisión de la cultura, la concepción que tenemos del ciberespacio es determinante para la construcción del mismo. Para poder ser parte activa de este proceso, no debemos obviar las inercias que los esquemas mentales predigitales nos producen a la hora de pensar en lo que la tecnología nos puede aportar, así como el inmovilismo que sufrimos ante una innovación cuando afecta a modelos de comportamiento asentados en nuestra cultura. Además, hemos de ser conscientes de que existen distintas visiones e intereses sobre cómo debe ser el ciberespacio. Actualmente se está dando una fuerte pugna por la definición, control y explotación de la Red entre las grandes operadoras de telecomunicaciones, las multinacionales tecnológicas de servicios y los movimientos por la neutralidad de la Red, el software libre y los contenidos abiertos.

Afortunadamente, el diseño y definición de la Red están abiertos y lo más interesante es que la plasticidad de las tecnologías digitales hace posible que sean los usuarios los que estén construyéndolas, desarrollando aplicaciones, servicios y contenidos, pero, sobre todo, expandiendo nuevas formas de comunicación. En este sentido, el desarrollo de la Web 2.0 posibilitó que se dejaran atrás modelos de interacción hombre-máquina, donde el componente tecnológico marcaba y guiaba de manera determinista 
las posibilidades de interacción entre los usuarios, y que se pasara a un modelo donde el componente humano -el estar con otros- cobra protagonismo y donde la tecnología permite que las propias relaciones sociales que surgen de la comunicación e interacción desarrollen cada vez más espacios virtuales que producen su propia cultura. Por tanto, estamos ante algo que va más allá de la idea de medio de comunicación, estamos ante una tecnología sobre la que se está construyendo un nuevo espacio antropológico (2): el ciberespacio.

Para Pierre Lévy, el ciberespacio o la Red es "el nuevo medio de comunicación que emerge de la interconexión mundial de los ordenadores. El término designa no solamente la infraestructura material de la comunicación numérica, sino también el oceánico universo de informaciones que contiene, así como los seres humanos que navegan por él y lo alimentan" (Lévy 2007: 1). Destacamos el hecho de que el ciberespacio contiene al usuario participante, siendo su papel determinante, pues, como afirma Mayans (2003), "el ciberespacio sólo es, sólo existe, en tanto que estemos ahí. Pero no sólo para escucharlo, sino en este caso también para crearlo, en toda su extensión. El ciberespacio no es una red de ordenadores, sino el resultado de la actividad social de los usuarios y usuarias de los ordenadores conectados entre sí que se reparten -desigualmente, eso sí- por todo el mundo".

Esta actividad social está sometida a las propiedades consustanciales a la naturaleza del nuevo espacio que contrastan con el espacio físico. El primer elemento diferencial es la materia prima con la que se construye: la información. La información constituye el recurso esencial de construcción, que a nivel físico consiste en secuencias de bits, en forma de impulsos eléctricos o marcas en las memorias informáticas, pero que a nivel ciberespacial se convierten en objetos de información que, como si de ADN se tratara, contendrán las instrucciones necesarias para poder actualizarse en una aplicación, texto, imagen, vídeo, música, etc. Por otro lado, estos objetos no están sometidos a los principios de finitud del espacio físico; es decir, aunque existen límites en la capacidad de almacenamiento de bits de los dispositivos, desde el punto de vista ciberespacial no existe restricción material, pues la información puede expandirse infinitamente y los objetos pueden replicarse sucesivas veces de manera perfecta. En muchos casos, solo la invocación o petición desde cualquier punto de acceso generará una copia o una actualización en el terminal del usuario y esto no supone consumo de una materia finita. En este espacio de la abundancia, las limitaciones de replicación solo pueden imponerse de manera externa.

Otro elemento diferencial del ciberespacio es una redefinición de las dimensiones espacio y tiempo. Aunque la información está almacenada en algún lugar de la infraestructura y, por tanto, cuenta con una ubicación espacial concreta del mundo físico, se puede acceder a la información desde cualquier punto de acceso sin percepción de su emplazamiento, por lo que, en sentido ciberespacial, la distancia no existe. Por su parte, el tiempo es modular, una interacción comunicativa entre los sujetos participantes puede darse de manera instantánea o ralentizada mediando lapsos de tiempo significativos entre cada intervención, sin que ello suponga una merma o imposibilidad para que esta se desarrolle plenamente.

Estructuralmente, por su propia definición, la Red es distribuida, carece de centro, lo que ha facilitado un alto grado de horizontalidad en su desarrollo. Aunque es factible la comunicación uno a muchos -como los modelos broadcasting de los massmedia-, sobresalen las tecnologías que facilitan la interacción de muchos a muchos, donde no existe un emisor privilegiado ni una audiencia monolítica, sino que todos son potencialmente emisores y receptores, un fenómeno que ha alterado la estructura de poder al abrirse nuevas vías de gestión de la información y construcción de conocimiento. Además, la Red puede albergar todo tipo de información y posibilita su intercambio entre cualquier usuario. En este sentido, la infraestructura de comunicaciones es neutral respecto a los contenidos que por ella circulan y respecto a la identidad de las personas que interactúan.

Sobre estas propiedades se construyen los espacios de participación. Pero ¿supone esta participación una mera habilidad técnica o se trataría de una nueva cultura? Es decir, ¿cambian los valores, creencias, el lenguaje, las formas de conocimiento, de creación, las tradiciones y modos de vida de los que participan? Resulta obvio que el ciberespacio ha producido una nueva cultura: la cibercultura. Para Lévy, la cibercultura designa "el conjunto de las técnicas (materiales e intelectuales), de las prácticas, de las actitudes, de los modos de pensamiento y de los valores que se desarrollan conjuntamente en el crecimiento del ciberespacio" (Lévy 2007 y 2011). Si en la visión clásica de cultura eran clave los aspectos simbólicos (ideas, valores y normas), la cibercultura incluirá además como aspectos fundamentales los artefactos y las técnicas. Así, nuestra idea de cibercultura contemplará los medios simbólicos y materiales, y los efectos 
sobre las capacidades cognitivas individuales y colectivas, como la memoria, la percepción, el razonamiento, la imaginación y la creatividad.

La cibercultura se levanta sobre un axioma: "nadie lo sabe todo, todo el mundo sabe algo, todo el conocimiento está en la humanidad. No existe ningún reservorio de conocimiento trascendente y el conocimiento no es otro que lo que sabe la gente" (Lévy 2004: 20). Siguiendo a este autor, consideramos que existen tres principios que han orientado el crecimiento inicial del ciberespacio y las distintas expresiones de la cibercultura: la interconexión, la creación de comunidades virtuales y la inteligencia colectiva.

Estos principios han de ser cubiertos para poder alcanzar los máximos beneficios que el ciberespacio permite. El más elemental sería el principio de interconexión, fundado sobre el axioma de que la interconexión es mejor que el aislamiento. Se alcanza cuando tenemos posibilidad de acceso a las infraestructuras de comunicaciones desde el espacio geográfico en el que estamos y disponemos de la competencia mínima para iniciar una actividad. Una vez cubierto este paso básico, surge la necesidad de participar en comunidades virtuales, pasamos entonces a ser miembros activos que interactúan, produciéndose una socialización que, adecuadamente desarrollada, puede constituir una experiencia de ciudadanía digital.

Las distintas formas de socialización varían en función de la organización de las comunidades virtuales. Debemos distinguir entre las que se vinculan al territorio físico, virtualizando las instituciones y organizaciones (por ejemplo, la escuela o la clase; en tal caso, no se amplía significativamente la interconexión entre los sujetos, pero sí las posibilidades de interacción entre ellos y con la información) y las que se organizan transcendiendo las limitaciones del territorio físico, constituyéndose sobre algún interés compartido. Un elemento importante de estas comunidades tiene que ver con la aceptación de la interconexión total o restringida, lo que se traduciría en comunidades abiertas o cerradas. En el caso de la virtualización del territorio, las posibilidades de interconexión ya estarían definidas, no así en las comunidades organizadas por intereses. Como podemos observar, la cuestión abierto/cerrado constituye un nuevo tipo de frontera basada en una multiplicidad de casuísticas que vendrán definidas desde la propia comunidad.

Las posibilidades de interacción en las comunidades virtuales determinan el conjunto de situaciones sociales que se pueden dar, que solo podrán expresarse en cuanto el software lo permita y de la forma que lo regule. Por tanto, el diseño de este software deberá tener en cuenta consideraciones sobre la interacción social y las posibilidades que pueden emerger de la comunicación y colaboración dentro del grupo. La gestión de situaciones sociales como el spam, trolls, las discusiones fuera de lugar y otras muchas que surgen de la interacción social en el nuevo espacio se resuelven a través de soluciones tanto tecnológicas, que limitan o controlan la participación, como a través de sistemas de autorregulación social de la propia comunidad, a través de la construcción de reglas de comportamiento (netiquetas) que los participantes han de cumplir. Los propios participantes manejan las intervenciones que se desvían de los usos a los que se orienta el servicio. El propio grupo sabe que tiene que autorregularse para conseguir un espacio acorde con sus objetivos y donde se pueda desarrollar una interacción y comunicación que vaya construyendo una comunidad cohesionada y enriquecedora para todos (Rhoderick 2013).

En definitiva, el principio de organización de los usuarios en comunidades virtuales requiere definir tres aspectos: el primero, un diseño del software social que acoge la comunidad coherente con la actividad que se va a desarrollar; segundo, los límites de la comunidad, si está vinculada al territorio o no, y si es abierta o cerrada; y en tercer lugar, el desarrollo de unas reglas, normas e incluso microcultura que rijan la vida de la comunidad en aras del mejor aprovechamiento de la inteligencia colectiva, finalidad última de toda comunidad.

Pero ¿qué es realmente la inteligencia colectiva? Lévy (2004) la define como una forma de inteligencia universalmente distribuida, constantemente realzada, coordinada en tiempo real, y resultando en la movilización efectiva de habilidades. La base y meta de inteligencia colectiva es el reconocimiento mutuo y enriquecimiento de los individuos. Desde el Center for Colletive Inteligence del MIT nos ofrecen una definición más operativa de inteligencia colectiva: "grupos de personas que hacen cosas colectivamente de manera inteligente" (Malone 2012). Para estos autores, la inteligencia colectiva se ha dado desde las sociedades cazadoras-recolectoras; lo que es novedoso en la actualidad es el ciberespacio y lo que hay que conseguir es resolver la cuestión de cómo, a través del ciberespacio, podemos ser y actuar de manera 
más inteligente. Acertadamente determinan que no es suficiente la interconexión, pues es necesario explotar de manera adecuada el principio de organización y crear sistemas que maximicen la inteligencia colectiva.

Los sistemas para la explotación de la inteligencia colectiva pueden darse de manera centralizada, a través de plataformas donde se establecen las posibilidades de organización e interacción, que requerirá cierta coordinación central, o a través de sistemas distribuidos, donde las herramientas son de gestión individual o de pequeño grupo, pero interconectadas, formando redes. En ambos casos, los miembros de la comunidad se unen libremente para la creación de bienes comunes, que quedarían a disposición de todos. Esta forma de colaboración funcionaría según un modelo de producción P2P (3) (Bauwens 2005a, 2005b y 2009), en el que el procomún es la fuente de conocimiento y los recursos de la comunidad, y todo lo producido a partir de él es devuelto al procomún, que se desarrolla de manera ilimitada.

Aunque resulte evidente, no podemos obviar que un ciberespacio vacío carece de valor, por lo que habría que llenarlo, darle vida. El punto de partida del ciberespacio es el procomún: software, aplicaciones, plataformas, contenidos, datos, metadatos, redes sociales, comunidades, etcétera. Por tanto, para comprender el encuentro de las tecnologías digitales y la educación es necesario analizar el procomún educativo, desvelar las creencias que impulsan su desarrollo y las formas de participación de los actores educativos.

\section{Abordaje del problema $\underline{(4)}$}

Para abordar nuestro problema, coincidimos con Hine (2004) en la necesidad de considerar Internet como artefacto cultural y también como cultura. Esto nos obligó a repensar la relación de ciberespacio y etnografía. En nuestra investigación, la metodología etnográfica ha sido enriquecida con técnicas de análisis de sistemas de inteligencia colectiva. Las técnicas etnográficas clásicas nos permitieron esclarecer qué ocurre a través de la visión de los actores, sus experiencias, sus expectativas, las dificultades que encuentran y las acciones que realizan. La técnica para el estudio de los sistemas de inteligencia colectiva que empleamos se basa en una metodología de análisis específica desarrollada de manera inductiva, a partir del estudio de cientos de sistemas, donde los autores consiguieron identificar los bloques de construcción básicos, que, siguiendo una metáfora biológica, denominaron "genes" (Malone 2009). Así, los sistemas de inteligencia colectiva se pueden caracterizar por su "genoma", es decir, por la combinación de genes que los constituyen. Cada gen es útil bajo determinadas condiciones, por lo que la selección de estos y sus posibles combinaciones es crucial para aprovechar de manera efectiva el poder de las multitudes, en definitiva, potenciar la interconexión entre las personas y optimizar el principio de organización de la comunidad para conseguir el mejor aprovechamiento de la inteligencia colectiva. La virtud de este método es que permite ordenar el análisis de los sistemas utilizados, averiguar los genomas y determinar si los genes empleados son los más adecuados. A partir de esta información, pudimos deducir los a priori del diseño, creencias y valores que guiaron su construcción.

Determinar los sistemas de inteligencia colectiva disponibles orientados a educación y valorar su impacto es una tarea fundamental. Una exploración de la web nos muestra que la diversidad de sistemas destinados o utilizados para fines educativos resulta inabarcable, por lo que optamos por seleccionar los más usados en los centros investigados. Una vez recopilados, los organizamos atendiendo a su objetivo principal. Aunque algunos, como la blogosfera, se utilizan para distintos fines, la mayoría se orienta hacia uno de los siguientes ámbitos: contenidos educativos abiertos o desarrollo profesional docente.

Los sistemas de inteligencia colectiva sobre contenidos educativos abiertos más utilizados en los centros son: Wikipedia, Youtube, Blogosfera educativa y los portales institucionales de contenidos. Los portales institucionales son una iniciativa generalizada en todas las CC.AA. y cada una gestiona su propio espacio de recursos. Asimismo, el Ministerio de Educación promueve portales de contenidos propios y repositorios específicos en coordinación con las administraciones autonómicas, como el proyecto Agrega, una de las mayores inversiones realizadas para el desarrollo y difusión de contenidos educativos.

El segundo conjunto de sistemas de inteligencia colectiva se centraría en las redes profesionales docentes para el intercambio de experiencias, apoyo, colaboraciones y aprendizaje entre iguales. Si en el caso de los contenidos encontramos ciertas regularidades en el uso de algunos sistemas, en el caso de las redes 
profesionales prima la diversidad. El profesorado participa en multitud de sistemas sobre temas genéricos o muy especializados, en grandes comunidades o pequeños grupos. Algunos casos relevantes son: la blogosfera educativa, la comunidad "Internet en el aula", departamentos virtuales de algunas CC.AA. y redes de profesores por especialidad.

Hemos buscado con insistencia sistemas dirigidos específicamente a la participación del alumnado y hemos comprobado que son escasos. A gran escala, el más conocido sería Etwining, una comunidad de centros educativos europeos orientada al encuentro, reflexión, intercambio de conocimiento y creación de proyectos entre profesores y alumnos de distintos países de la Unión europea. Y, a pequeña escala, algunos profesores organizan actividades virtuales entre clases, del mismo o distinto centro, apoyándose en las plataformas propias de sus centros, redes sociales genéricas, blogs o wikis.

Al construir el genoma de estos sistemas y analizarlos a la luz de la metodología propuesta por Malone, alcanzamos los a priori del diseño, las creencias que los sostienen y su potencial para explotar la inteligencia colectiva. Pero, además, estos sistemas nos interesan como base o lugar de asentamientos de comunidades en el ciberespacio, donde se resuelve una nueva forma de "vida". En este punto recurrimos a técnicas de etnografía virtual; en concreto, la observación virtual, que sería la observación sobre la producción de recursos, soluciones y servicios que se ubican en Internet, pero también de los fenómenos que ocurren en la interacción social mediada por esta tecnología (lkegami 2011 y Breslow 2012). La observación ha sido participante en el caso de las comunidades virtuales creadas en las plataformas de centro, en la comunidad "Internet en el aula", departamentos virtuales y redes de profesores por especialidad. En algunos casos la configuración de los espacios en abierto, como es el caso de los blog, haciendo público lo que en ellos se dice o se discute, ha permitido analizar estas interacciones como mero observador.

\section{Asentamientos educativos en el ciberespacio}

Del análisis de los sistemas diseñados por las administraciones para la gestión de contenidos, encontramos un rasgo común y limitante: la centralización. El primer nivel de centralización se encuentra en las infraestructuras, de donde parte el poder para aplicar el segundo nivel, que estaría en la selección de lo que es publicable y lo que no, lo que los aleja de los modelos seguidos por los sistemas de mayor éxito que se han dado en internet para la gestión de contenidos abiertos, como Youtube o Wikipedia. Se trata de que la multitud de usuarios haga el trabajo, lo que resulta, como vemos en estos proyectos de éxito, económico y eficaz. Entonces, ¿por qué la administración no aplica estas fórmulas? Creemos, siguiendo a Malone, que los políticos, asesores, gerentes y técnicos que intervienen en el desarrollo de estos sistemas no saben cómo hacerlos. Se necesita un mayor conocimiento sobre cómo diseñarlos para que el potencial de la inteligencia colectiva se desate. Pero, en nuestra opinión, además, se requiere superar algunas creencias limitantes, muy extendidas en el ámbito educativo; en concreto, la creencia en la necesidad del canon, en la jerarquía y en el principio de escasez.

La creencia en la necesidad de un canon implica que solo los recursos de alta calidad deben ser publicados, el resto se considera inservible y se desecha. En coherencia con esta creencia, todos los sistemas institucionales se rigen por un estricto sistema de selección y control de calidad. Sin embargo, si consideramos que la información se revaloriza por el flujo, readaptación y modificación constante, esta forma de actuación desaprovecha una gran cantidad de recursos y conocimiento que pueden ser altamente valiosos.

A esta creencia hay que añadir la pasión por la jerarquía que las administraciones educativas tienen, tanto para establecer el canon según los estándares de selección propuestos por sus "expertos", como para garantizar los derechos de copyright. Las escrupulosidad en la gestión de los derechos de lo que se publica en sus plataformas y portales supone una centralización limitante, que, como podemos ver en otros sistemas, se puede superar articulando modelos donde el propio grupo avisa o denuncia materiales inadecuados y cada publicador se responsabiliza de sus contenidos. Si la creencia en el canon cambiara y se superara la pasión por las jerarquías supervisoras y valedoras de lo que es apropiado, podría publicarse todo el material disponible o generado; sin embargo, aún habría que superar otra creencia: el principio de escasez. 
El principio de escasez ha guiado las formas de publicación tradicionales, que seguían el principio de Pareto o proporción de $80 / 20$, es decir, centrarse en el $20 \%$ de los productos y usuarios que proporcionan el $80 \%$ del beneficio, lo que tiene sentido en el mundo físico por los costes de producción, almacenamiento y distribución, en el ciberespacio se convierte en una creencia limitante. El ciberespacio es el lugar de la abundancia, por tanto, los sistemas de inteligencia colectiva pueden almacenar de manera adecuada toda la información disponible, colocando en primer plano lo más relevante, pero sin despreciar el resto. Cualquier recurso puede ser interesante para alguien que lo reutilizará y lo pondrá de nuevo en el conjunto de contenidos abiertos, favoreciendo el flujo y la creación constante.

Mientras estas tres creencias no se superen, los sistemas diseñados no explotarán todo el potencial de la inteligencia colectiva, por lo que se desaprovechará el conocimiento y el dinamismo de la multitud de creadores. Hay que destacar que el uso de los sistemas creados por las administraciones es bastante bajo. Muchos de los repositorios que hemos estudiado son desconocidos por la mayoría del profesorado, y los que han accedido a ellos pocas veces los consideran útiles. No parece que los esfuerzos y planteamientos de la administración se hayan traducido en un mejor aprovechamiento. Podemos afirmar que los sistemas de inteligencia colectiva más utilizados en los centros no son los institucionales centralizados, con materiales y recursos organizados y seleccionados por expertos, sino los recursos publicados de manera individual por la gran masa de participantes en el ciberespacio.

El profesorado desarrolla sus propios modelos de apropiación, explotación e incluso de diseño de proyectos, apoyándose normalmente en webs, blogs, wikis, plataformas y servicios de almacenamiento de vídeos, audios, documentos, presentaciones, etc. Al analizar estas iniciativas surgidas desde abajo, comprobamos que son sistemas que aprovechan las aportaciones de la multitud sin aplicar jerarquías de poder sobre lo publicable. Todas las aportaciones son valiosas y tienen su lugar en el sistema, de ahí que sean los propios participantes los que con sus votos, comentarios, enlaces, etc., dan valor a una participación o a un contenido. Estos sistemas se pueden basar en infraestructuras centralizadas (plataformas) o distribuidas (blogosfera y red de PLE (5)), pero en todos los casos el modelo de producción es P2P, donde los participantes, individualmente y de manera horizontal, participan en la creación y autogestión del conocimiento.

Las plataformas implican una gestión técnica y administración centralizada, aunque la gestión social la realiza la comunidad. En el caso de sistemas distribuidos, cada participante administra de manera autónoma su propio espacio. Sin duda alguna, en este momento, la blogosfera constituye una de las tecnologías más decisivas en el desarrollo del ciberespacio, surge por evolución espontánea a través de las aportaciones individuales y distribuidas del conjunto de blogs que se interrelacionan, se citan y enlazan, favoreciendo la elaboración y reelaboración de la información y el conocimiento en un flujo incesante. No debe sorprendernos que la blogosfera educativa sea el sistema de inteligencia colectiva con mayor impacto en educación, pues es la tecnología que más se acerca a los principios del ciberespacio: abundancia, distribución, flexibilidad, horizontalidad, libertad... Los blogs y, por extensión, los PLE conforman redes totalmente distribuidas; no existe un centro privilegiado desde donde controlar y decidir qué merece la pena y qué no. El individuo es el centro, lo que él crea y construye es relevante para él y es accesible para quien quiera seguirlo y aprovecharlo. La participación alcanza su máxima expresión, ya que grupos de individuos pueden compartir, debatir y colaborar desde el principio de igualdad en una estructura horizontal y abierta.

\section{La "vida” en el ciberespacio}

Cuando alguien interesado en un tema no encuentra en su entorno personas con los mismos intereses, puede fácilmente integrarse o desarrollar una comunidad virtual sobre el tema que le preocupa. En ella, lo que antes era soledad se convierte en acompañamiento y posibilidad de desarrollar un espacio para conversar, crear y compartir con otros. Al mismo tiempo, el reconocimiento y las posibilidades de participar en la construcción de algo con valor no solo resulta sumamente atractivo, sino que es un estímulo para permanecer.

La actividad social que desarrolla la comunidad está condicionada por la organización del sistema de inteligencia colectiva. En las plataformas, las comunidades están más definidas. Al ingresar, se asume la meta compartida y la red social existente. En los espacios personales como blogs y PLE, la finalidad y los vínculos sociales los define exclusivamente cada creador, desarrollando su comunidad desde cero de 
manera completamente libre, una comunidad que evolucionará según los propios intereses. En cualquier caso, las comunidades virtuales se caracterizan por la diversidad de sus miembros, pues en su conformación no importa la edad, el sexo o la procedencia, sino el interés por el tema que los une. Al centrarse el vínculo social de la comunidad en un interés compartido y no en filiaciones personales, como ocurre en las comunidades de nuestra vida fuera de la pantalla, abandonarlas se realiza de manera rápida y sin presiones. De esta forma, comprobamos que la organización por intereses y la facilidad para abandonar favorecen la homogeneidad respecto a opiniones y creencias entre los miembros. Por lo general, no se producen debates o controversias críticas o divergentes y las interacciones son fundamentalmente para distribuir información que refuerza y consolida la opinión del grupo. En otra palabras, el recién llegado que explora las interacciones no pretende debatir ni intentar convencer; por el contrario, si los planteamientos no se ajustan a su línea de pensamiento, resulta más sencillo abandonar la comunidad y encontrar otra acorde con sus ideas.

El producto o conocimiento generado en estas comunidades se transfiere a lo local a través de los profesores participantes, que se convierten en puentes entre las comunidades virtuales y sus centros, haciendo que cada vez sea más difícil que las buenas experiencias lejanas, materiales e ideas puedan quedar ocultos o inalcanzables. El resultado de los sistemas de inteligencia colectiva terminan repercutiendo a escala global: un buen material educativo, una metodología, el tratamiento del alumno con necesidades especiales, etc., queda abierto en el ciberespacio y accesible para cualquiera. Pero, en este proceso de expansión de la inteligencia colectiva, son los participantes en comunidades virtuales los primeros en conocer la innovación, captar el beneficio, integrarlo en sus clases y proponer los cambios a sus pares y en sus centros.

La propagación de las innovaciones es más eficiente a través de los sistemas de explotación de la inteligencia colectiva que a través de los sistemas tradicionales. Esta situación genera dos velocidades en la asimilación y adaptación del profesorado a nuevas ideas. Es bastante común que los planteamientos innovadores del profesorado que actúa de puente entre las comunidades virtuales y el centro choque con resistencias, fruto de esta brecha de conocimientos y visiones, a lo que se sumaría la rigidez organizativa de los centros. El resultado es que la innovación pasa de la comunidad virtual a la clase sin afectar a la escuela, que escasas veces se ve transformada.

Para comprender los matices de esta brecha, debemos considerar que, aunque existe una gran comunidad educativa participando en el ciberespacio, en términos absolutos, es un porcentaje muy pequeño de la comunidad real, a lo que se suman distintos grados y formas de participar. En estos momentos, la práctica totalidad del profesorado busca y recopila información en la Red para sus clases, pero son una minoría los que participan en sistemas de inteligencia colectiva, produciendo información o recursos para el procomún. No obstante, hemos observado un aumento de la participación en microredes cerradas, especialmente a través de correo electrónico y listas de distribución, y cada vez más en comunidades de centro, aulas virtuales y cursos de formación en línea. El interés por los espacios personales estuvo desarrollándose desde el 2000, pero se estancó a finales del 2007, cuando la atención de los medios tradicionales y de la propia Red se desplazó hacia redes sociales tipo Facebook y al desarrollo de plataformas educativas. En estos años, los centros y las administraciones han fomentado el desarrollo de la virtualización de aulas, departamentos y otros órganos de los centros. Estos nuevos espacios facilitan la comunicación entre los miembros de la comunidad educativa de cada centro y consiguen cierta experiencia de inmersión del alumnado en comunidades protegidas; sin embargo, se han convertido en las cavernas del profesorado, donde se da un alto nivel de actividad y recursos que no son visibles y que no aportan valor al procomún.

Si toda esta actividad se diera de manera abierta, se desencadenaría un desarrollo de la inteligencia colectiva sin precedentes. Nos preguntamos: ¿por qué el profesorado no participa de manera abierta?, ¿por qué se mantiene en estas cavernas virtuales? No hay una respuesta única. Se trata de un conjunto de comportamientos, creencias y actitudes que la mayoría de las veces se superponen. En primer lugar, es necesario destacar que está muy instaurada la cultura de la fotocopiadora, ahora del "copia y pega". El profesorado maneja para sus clases excelentes materiales elaborados a partir de libros e imágenes con restricciones de copyright, sin consideraciones sobre las fuentes y la autoría. Esto hace que los materiales se usen en sus clases, pero no se entreguen al procomún, aunque excepcionalmente algunos han dado un salto cualitativo, creando blogs bajo una identidad anónima para poner al alcance no solo de sus alumnos, sino de todo el mundo, estos contenidos educativos, lo que lleva a sus últimas consecuencias el modo de producción P2P y la cultura hacker. En segundo lugar, observamos una creencia limitante: muchos 
profesores consideran que lo que saben o crean no tiene suficiente valor para ser compartido. Además, siguen pensando que el material se ha de publicar bien elaborado, cuando lo cierto es que en los nuevos entornos cualquier material es un punto de partida para alguien y puede ser mejorado de manera iterativa por la comunidad. Por último, hay que destacar que el profesorado comparte actuando por reciprocidad: da generosamente materiales, recursos o ayuda a cualquier compañero que lo necesite, esperando un retorno directo, que puede ser el simple agradecimiento o una ayuda concreta en el futuro. No está instaurada la cultura P2P y no se ha interiorizado el valor del bien comunal donde dar y recibir no sigue las reglas de reciprocidad habituales, pues desconocemos quién puede aprovechar lo que entregamos y tomamos lo que necesitamos sin establecer obligaciones o deudas de reciprocidad.

Sin duda, son muchas las formas de estar en el ciberespacio y por supuesto compatibles, pero destacaremos dos que reflejan los extremos del espectro de posibilidades: la primera y más extendida vería el ciberespacio como fuente de información y medio para mejorar la comunicación de los actores de la institución gracias a la virtualización de aulas, departamentos, claustro y otros órganos formales del centro (en estos casos, la actividad social y educativa se da en entornos o redes cerrados); y la segunda, lo contemplaría como espacio humano abierto, organizado por temas de intereses y proyectos colectivos, donde damos y recibimos a través de una reciprocidad a veces directa, pero sobre todo indirecta, a través de redes $\mathrm{P} 2 \mathrm{P}$ y el conjunto de bienes comunales. Bajo este modelo, se transciende lo local e individual para alcanzar lo global y colectivo, abriendo la clase y la escuela al nuevo espacio, siendo miembro de él, organizándose en comunidades lo más abiertas posible para aprovechar y expandir la inteligencia colectiva.

Cada modelo genera una experiencia distinta de ciudadanía digital y, por extensión, de competencia digital. No podemos ignorar que solo el profesorado ya socializado en sistemas de inteligencia colectiva es capaz de acompañar al alumnado, generando espacios adecuados para que desarrolle experiencias significativas de ciudadanía digital. Cada paso en ese sentido es un avance de la educación en la colonización del ciberespacio y, por tanto, de expansión y desarrollo de nuestra inteligencia colectiva desde valores educativos. Las consecuencias son experiencias que sobrepasan el ámbito local y que transforman las relaciones y valores tradicionales de grupo, territorio, nación, etnia, organización clasista, conocimiento privado, por otros nuevos: unidad humana, organización por temas de interés, conocimiento abierto, modo de producción $\mathrm{P} 2 \mathrm{P}$ y cultura hacker, que están produciendo un procomún educativo que no cesa de crecer y que está a disposición de la escuela, la clase, el profesado y el alumnado de todo el mundo. Sin embargo, esta forma ser, sentir, percibir, pensar y estar en el ciberespacio no alcanza a todo el alumnado. La realidad nos muestra que la mayoría de los alumnos no tienen oportunidad de experienciar estos nuevos valores, su socialización en el ciberespacio se realiza de manera exclusiva a través de la participación en multitud de redes sociales, clubs de fans, juegos en red y servicios de moda salvajemente condicionados por intereses empresariales, inundados de publicidad inadecuada y estrategias de captación de clientes que siguen esquemas de negocio en muchos casos poco éticos o delictivos.

\section{De la integración a la "eduagorización"}

En la literatura científica, "integración" es la categoría fundamental desde la que se aborda el encuentro de las TIC y la educación. Desde un punto de vista formal, el término "integrar" tiene el sentido de aunar entidades divergentes entre sí para crear un todo que las sintetice. En el caso que nos ocupa, integrar las TIC en educación puede producir un enriquecimiento de la educación, la escuela y la clase, sumando aquellas tecnologías que la potencian o puede ir más allá y producir una transformación que altere sustancialmente la forma educativa. Entre la comunidad educativa, está muy extendida la idea de que integrar las TIC equivale a introducir la tecnología en las clases y las escuelas para mejorarlas, enriqueciéndolas pero sin transformarlas, dejando marginadas las posibilidades que tienen las actuaciones en el ciberespacio para desarrollar la inteligencia colectiva.

Es incuestionable que la concepción que la comunidad educativa tiene del ciberespacio es determinante en la construcción del mismo y en las aportaciones que este puede hacer a la educación. Por ello, resulta crítico cambiar o ampliar el concepto de "integración" para poder captar cognitivamente todas las dimensiones y matices del encuentro TIC y Educación. En nuestra opinión, necesitamos una nueva categoría con entidad propia y equiparable a la visión más extendida de "integración" que permita concebir y revalorizar los procesos de explotación de la inteligencia colectiva. Proponemos llamarla "eduagorización", acción y efecto de "eduagorizar" (6), o crear ágoras educativas, asentamientos capaces de acoger comunidades que se 
organicen para el desarrollo de la inteligencia colectiva.

Como consecuencia, hemos de reconsiderar el desarrollo del encuentro TIC-educación, para verlo bajo estas dos categorías: integración y eduagorización. Estos procesos se complementan creando sinergias con un enorme potencial de transformación. El primero, integrando las TIC cada vez más en los distintos ámbitos educativos (esta es la apropiación más evidente, que se ajustaría a la visión más extendida); y, la segunda, a través de la expansión de la educación por el ciberespacio, creando asentamientos que desarrollan la inteligencia colectiva.

Para precisar lo que significa "expandir la educación por el ciberespacio", hemos de recuperar la concepción de ciberespacio como espacio antropológico y ver el fenómeno como una metáfora de colonización, donde colonizar implica crear asentamientos permanentes y abiertos, tener presencia y actuar colectivamente en la generación de bienes comunales. Pasar por el ciberespacio, establecer una caverna o gueto cerrado para una actividad y desmantelarlo no es colonizar. Los asentamientos cerrados o temporales como forma de ampliar el espacio y el tiempo de una escuela o clase para sí misma serían una forma de integración al no haber aportación al procomún.

En consecuencia, mientras la integración de las TIC intenta potenciar y mejorar el mundo educativo tal como lo conocemos, favoreciendo el desarrollo humano individual en el ámbito local del aula y de la escuela, la eduagorización aprovecha el ciberespacio para transcender este ámbito y permitir una experiencia educativa más allá de la clase y de la escuela, permitiendo el desarrollo no solo del procomún, sino también de la competencia digital del alumnado desde esta nueva perspectiva.

\section{Conclusión}

El ciberespacio está facilitando nuevas formas de interacción, aprendizaje y creación. Los sistemas actuales despliegan posibilidades diversas de organización y explotación de la inteligencia colectiva. Observamos que los sistemas que articulan una organización horizontal y abierta suponen la opción de mayor impacto sobre el aprendizaje y la creación de conocimiento. Esta forma de organización permite la autogestión del saber y el conocimiento partiendo del procomún, que es la fuente desde la que crear e innovar y donde vuelven todas las nuevas creaciones, en un ciclo constante de innovación y desarrollo que expande nuestra inteligencia colectiva.

Sin embargo, las estructuras emergentes tienden a modelarse bajo los patrones tradicionales de interpretación de la realidad, a veces incluso forzando la propia naturaleza de la Red para dar continuidad a las normas, creencias, valores, instituciones y formas sociales establecidas. En este sentido, observamos que los sistemas creados por las administraciones educativas no han alcanzado los objetivos marcados; de hecho, la mayoría de los profesores no los utiliza y, en muchos casos, ni ha oído hablar de ellos. Consideramos que estos sistemas han de revisarse a la luz del nuevo conocimiento disponible sobre los sistemas de explotación de inteligencia colectiva, lo que implica superar aquellas creencias que limitan el aprovechamiento de las propiedades del ciberespacio. Actualmente, la mayoría de los sistemas institucionales son centralizados, basados en la escasez, el canon y la jerarquía, principios totalmente divergentes de los sistemas de inteligencia colectiva de mayor éxito en internet y contrarios a la naturaleza del ciberespacio. Además, la mayoría están orientados a proporcionar contenidos cuando realmente ya no es tan urgente ahondar en grandes fuentes o repositorios de información, pues existe una sobreinformación. Por el contrario, lo que se requiere es una mayor participación humana en la gestión de la información, para transformarla en conocimiento y sabiduría.

Los sistemas de inteligencia colectiva más eficaces son los que propician la construcción de comunidades donde las personas ya no entran, buscan, cogen y salen de manera anónima, sino que permanecen para conversar, crear y compartir, "estando" con otros y habitando un espacio donde son reconocidos. Estas comunidades se desarrollan bajo nuevos principios y valores: unidad humana, organización por temas de interés, conocimiento abierto, modo de producción $\mathrm{P} 2 \mathrm{P}$, cultura hacker y formas de dar y recibir sin interés económico y sin esperar una reciprocidad directa, pues entregamos sin saber a quién y tomamos sin deber a nadie, es el procomún la fuente y el destino de toda participación.

En educación, este proceso se está dando entre algunos profesores que participan activamente en 
comunidades profesionales y que han generado sus propios espacios personales de aprendizaje. Esta experiencia les permite crear actividades educativas ciberespaciales facilitando a sus alumnos interiorizar otra forma de ser, estar y pensar el ciberespacio. Sin embargo, son una minoría, pues la mayoría usa las TIC como fuente de información o medio de transmisión y los alumnos se socializan en el ciberespacio sin el acompañamiento de sus profesores o, en el mejor de los casos, a través del sucedáneo del aula virtual cuando es utilizada como espacio social y no exclusivamente para colgar apuntes y subir trabajos.

En nuestra opinión, no hemos sabido aprovechar el potencial del ciberespacio para la educación. Sin duda, existe un desconocimiento del nuevo espacio y de los mejores sistemas de explotación de la inteligencia colectiva que se pueden dar; pero también la concepción que los agentes educativos tienen de la integración de las TIC está condicionada, cuando no sesgada, por una visión inamovible del modelo actual de educación y escuela. En definitiva, un modelo predigital fuertemente anclado en nuestra cultura y en la manera de entender la educación, hasta el punto que integrar las TIC sigue siendo para la mayoría equivalente a mejorar la escuela y la clase tal como son, pero sin abrirse a los nuevos procesos de creación y gestión de conocimiento que se dan en el ciberespacio.

El camino para ampliar esta visión y revalorizar los principios del ciberespacio que son fundamentales para la educación de la sociedad de la información puede verse favorecido si los recogemos, tal como proponemos, bajo una nueva categoría. En consecuencia, el encuentro TIC y educación pasaría a ser abordado tanto bajo la perspectiva de la integración, propiciando el aprovechamiento de las TIC y el ciberespacio para la escuela y la clase, como desde el punto de vista de la eduagorización, expandiendo la educación por el ciberespacio de manera horizontal y abierta, desarrollando el conjunto de bienes comunales y la inteligencia colectiva humana. Ambos procesos, concebidos como paralelos y con valor equiparable, permitiría orientar las acciones de manera más equilibrada entre lo local y lo global, lo privado y lo común, el grupo y la totalidad humana, el conocimiento individual y la inteligencia colectiva, dando paso a una educación que aprovecha plenamente el ciberespacio y, por tanto, más cercana a lo que podría ser la forma educativa de la sociedad de la información.

\section{Notas}

1. Utilizaremos los términos "tecnología digital", "Tecnologías de la información y comunicación" o TIC de manera indistinta.

2. Siguiendo a Lévy (2004: 16), entendemos espacio antropológico como "un sistema de proximidad (espacio) propio del mundo humano (antropológico) y por consiguiente, dependiente de las técnicas, de las significaciones, lenguaje, la cultura, las convenciones, las representaciones y las emociones humanas."

3. P2P (peer-to-peer, en inglés) se refiere a redes de pares, entre iguales, o punto a punto. El concepto nace asociado a las redes de ordenadores donde cada nodo actúa simultáneamente como cliente y servidor respecto a los demás nodos de la red, permitiendo el intercambio directo de información entre cada nodo. En el contexto del nuevo modelo de producción, hace referencia a la superación de los modelos de escala; cada individuo o grupo local podrá, en una relación entre iguales, colaborar con otros en la consecución de un objetivo común.

4. Los resultados que presentamos forman parte de la investigación de tesis doctoral realizada entre 2007 y 2012. Durante los cursos $2007 / 2008$ y $2009 / 2010$, investigamos en escuelas, IES, centros de profesores y servicios dependientes de la Comunidad Autónoma de Murcia. En el curso 2010/2011, contrastamos los datos con centros de Cataluña y, durante el curso 2011/2012, con centros de Canarias, concretamente de Tenerife. Y, como continuidad de estos, nos abrimos al ciberespacio explorando redes sociales y espacios virtuales donde el profesorado y los alumnos de los centros investigados participaban.

5. PLE, acrónimo de "Personal Learning Environment", es Entorno Personal de Aprendizaje en español. Se trata de una agregación coherente de recursos y sistemas que el usuario gestiona personalmente, permitiéndole mantener un repositorio propio de información, espacios de publicación, reflexión y redes de 
contactos.

6. Eduágora es el nombre del prototipo de entorno social educativo o asentamiento ciberespacial que proponen Alberto Sierra y el autor del presente artículo. El término procede de la unión de "Educación" y "Ágora", pues, como el ágora griega, se trata de un espacio abierto y público (en nuestro caso, centrado en la educación). El entorno se concibe para que profesores, alumnos y otros agentes educativos de cualquier centro y territorio se organicen en comunidades para explotar la inteligencia colectiva, desarrollar el procomún educativo y permitir que los alumnos tengan la oportunidad de desarrollar una experiencia de ciudadanía digital a través de la inmersión en espacios de participación seguros más allá de su clase y de su centro (Moya 2010).

\section{Bibliografía}

Bauwens, Michel

2005a "The Political Economy of Peer Production", Ctheory.net.

http://www.ctheory.net/articles.aspx?id=499

2005b "P2P and Human Evolution: Peer to peer as the premise of a new mode of civilization", Institute of Network Cultures.

2009 "To the Finland Station, a 21st century update" Medialab-prado.

Breslow, H. (y Aris Mousoutzanis)

2012 Cybercultures: Mediations of Community, Culture, Politics. Amsterdam, Rodopi Press.

Cabrera, Jossue

2013 "Perspectivas teóricas de Cibercultura, y su aplicación en espacios educativos", Gazeta de Antropología, $\mathrm{n}^{\circ} 29$ (1) 8.

Hine, Christine

2004 Etnografía virtual. Barcelona, Editorial UOC.

lkegami, E.

2011 "Visualizing the networked self: agency, reflexivity and the social life of Avatars", Social Research, $\mathrm{n}^{\circ}$ $78 / 4$.

Lévy, Pierre

2004 Inteligencia colectiva: por una antropología del ciberespacio. Biblioteca virtual em Saúde.

http://inteligenciacolectiva.bvsalud.org/public/documents/pdf/es/inteligenciaColectiva.pdf

2007 Cibercultura: la cultura en la sociedad digital. Barcelona, Anthropos Editorial.

2011 Cibercultura: informe al consejo de Europa. Barcelona. Anthropos Editorial.

Malone, Thomas. (y otros)

2009 "Harnessing Crowds: Mapping the Genome of Collective Intelligence". MIT Sloan Research Paper, $\mathrm{n}^{\circ}$ 4732-09.

http://dx.doi.org/10.2139/ssrn.1381502

2012 Handbook of Collective Inteligent. MIT Center for Colletive intelligent. http://scripts.mit.edu/ cci/HCl/index.php?title=Main_Page

Mayans i Planells, Joan.

2003 "El ciberespacio, un nuevo espacio público para el desarrollo de la identidad local". Conferencia inaugural del III Encuentro de Telecentros y Redes de Telecentros, Peñafiel, Valladolid.

http://www.cibersociedad.net/archivo/articulo.php?art=158

Moya Juana (y otros)

2010 "Eduagora. Un entorno social educativo", en Francisco Martínez (y otros) (coord.), Tecnologías flexibles para espacios educativos diverso, Murcia, DM: (31-51). 
Rhoderick, N.

2013 "Exploring Cybercultures: Critical and Constructivist Studies on the Internet", Asia-Pacific Social Science Review, no 12/2. 